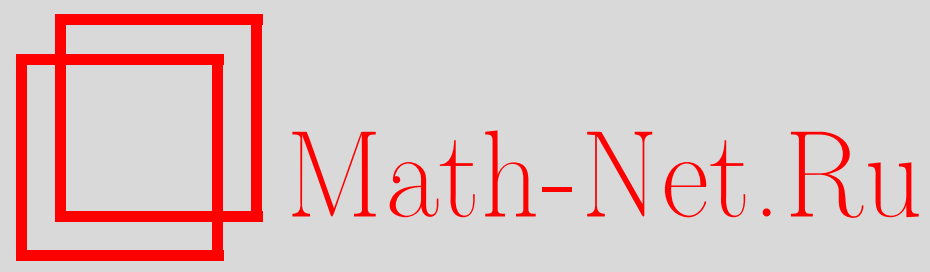

К. В. Костоусов, В. Т. Шевалдин, Аппроксимация локальными тригонометрическими сплайнами, Матем. заметки, 2005, том 77, выпуск 3, 354-363

DOI: https://doi.org/10.4213/mzm2498

Использование Общероссийского математического портала Math-Net.Ru подразумевает, что вы прочитали и согласны с пользовательским соглашением http://www . mathnet.ru/rus/agreement

Параметры загрузки:

IP: 54.237 .206 .68

26 апреля 2023 г., $12: 12: 42$

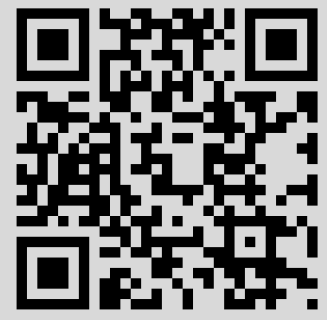




\title{
АППРОКСИМАЦИЯ ЛОКАЛЬНЫМИ ТРИГОНОМЕТРИЧЕСКИМИ СПЛАЙНАМИ
}

\author{
К. В. Костоусов, В. Т. Шевалдин
}

\begin{abstract}
Для класса $W_{\infty}^{\mathscr{L}_{2}}=\left\{f: f^{\prime} \in A C,\left\|f^{\prime \prime}+\alpha^{2} f\right\|_{\infty} \leqslant 1\right\} 1$-периодических функций построен неинтерполяционный линейный метод тригонометрической сплайн-аппроксимации, обладающий экстремальными и сглаживающими свойствами и наследующий локально свойство монотонности исходных данных (значений функции из $W_{\infty}^{\mathscr{L}_{2}}$ в точках равномерной сетки). Вычислена точно величина погрешности аппроксимации в равномерной метрике на этом классе. Она совпадает с величинами поперечников по Колмогорову и по Коновалову.

Библиография: 11 названий.
\end{abstract}

Введение. Численным аспектам апшроксимации действительных функций одного переменного, связанных с наследованием свойств монотонности и выпуклости исходных данных (чаще всего, это значения функции в точках равномерной сетки), посвящено значительное число работ (см., например, [1] и имеющиеся там ссылки). Однако, сохранение монотонности и выпуклости возможно, как правило, только при дополнительных довольно жестких ограничениях (см. ссылки в [1]); при этом, построенные процессы апшроксимации были, в основном, интерполящионного типа. На классе функций $W_{\infty}^{2}=\left\{f: f^{\prime} \in A C,\left\|f^{\prime \prime}\right\|_{\infty} \leqslant 1\right\}$ Ю.Н. Субботин [1] в 1993 году построил новый локальный неинтерполяционньй метод аппроксимации, использующий параболические сплайны с дополнительными узлами [2], сохраняющий указанные геометрические свойства исходных данных, и экстремальньй в смысле поперечников по Колмогорову и по Коновалову. В данной работе мы существенно используем идеи работы [1] и развиваем их для класса 1-периодических функций $W_{\infty}^{\mathscr{L}_{2}}=\left\{f: f^{\prime} \in A C,\left\|\mathscr{L}_{2}(\mathscr{D}) f\right\|_{\infty} \leqslant 1\right\}$. Здесь $A C$ - класс локально абсолютно непрерьвных функций, $\|\cdot\|_{\infty}=\|\cdot\|_{L_{\infty}[0 ; 1]}, L_{\infty}[0 ; 1]-$ класс функций, существенно ограниченных на $[0 ; 1]$, с обычным определением нормы

$$
\|f\|_{\infty}=\operatorname{ess}_{\sup } \in[0 ; 1]|f(x)|
$$

и $\mathscr{L}_{2}=\mathscr{L}_{2}(\mathscr{D})=\mathscr{D}^{2}+\alpha^{2} I \quad(\alpha>0, \mathscr{D}$ - оператор дифференцирования, $I-$ тождественньй оператор) - линейньй дифференциальньй оператор 2-го порядка с постоянными коэффициентами. За счет отказа от условий интерполяции в точках сетки для

Работа выполнена при финансовой поддержке Российского фонда фундаментальных исследований, грант № 02-01-00764, программы государственной поддержки "Ведущие научные школы", проект № НШ-1347.2003.1, и гранта поддержки научных исследований, выполняемых ученьми УрО $\mathrm{PAH}$ совместно с СО РАН. 
1-периодических функций нами построен линейный локальный метод тригонометрической сплайн-апшроксимации функций $f \in W_{\infty}^{\mathscr{L}_{2}}$, сохраняющий локально свойство монотонности и свойство, близкое к вьпуклости, исходных данных без каких-либо дополнительных ограничений на значения аппроксимируемой функции в узлах равномерной сетки. Метод использует тригонометрические сплайны (их еще называют $L$-сплайны; см., например, [3]) с дополнительными узлами, которые соответствуют дифференциальному оператору $\mathscr{L}_{2}=\mathscr{D}^{2}+\alpha^{2} I$, и обладает значительньпи преимуществами по сравнению с другими известными. В частности, на классе $W_{\infty}^{\mathscr{L}_{2}}$ построенньй метод является оптимальньм среди всех подпространств заданной размерности, а именно, величина погрешности апшроксимации функций $f \in W_{\infty}^{\mathscr{L}_{2}}$ в равномерной метрике посредством построенных тригонометрических сплайнов $S$, т.e. $\sup _{f \in W_{\infty} \mathscr{L}_{2}}\|f-S\|_{\infty}$, совпадает с величинами соответствующих поперечников по Колмогорову и по Коновалову. Для класса $W_{\infty}^{\mathscr{L}_{2}}$ были известны еще два таких экстремальных подпространства (см., например, [4] и имеющиеся там ссылки): тригонометрические полиномы заданного порядка и интерполящионные тригонометрические сплайны с равномерными узлами, однако эти линейные аппараты приближения не обладали формосохраняющими свойствами и свойством локальности в отличие от предлагаемого ниже метода.

1. Локальные тригонометрические сплайны. Пусть $\mathscr{L}_{2}=\mathscr{L}_{2}(\mathscr{D})=\mathscr{D}^{2}+\alpha^{2} I$ ( $\alpha>0, \mathscr{D}$ - оператор дифференцирования, $I$ - тождественный оператор),

$$
W_{\infty}^{\mathscr{L}_{2}}=\left\{f: f^{\prime} \in A C,\left\|\mathscr{L}_{2}(\mathscr{D}) f\right\|_{\infty} \leqslant 1\right\}
$$

- класс 1-периодических функций, $h=1 / n, n \in \mathbb{N}$, и $y_{j}=f(j h), j \in \mathbb{Z}$. Дифференциальному оператору $\mathscr{L}_{2}$ ставим в соответствие разностный оператор

$$
\Delta_{h}^{\mathscr{L}_{2}} y_{j}=\Delta_{h}^{\mathscr{L}_{2}} f(j h)=y_{j+2}-(2 \cos \alpha h) y_{j+1}+y_{j}
$$

определенный на пространстве последовательностей $\left\{y_{j}\right\}_{j \in \mathbb{Z}}$. Ясно, что если $f(x)=$ $\sin \alpha x$ или $f(x)=\cos \alpha x$ (функции из ядра оператора $\mathscr{L}_{2}$ ), то $\Delta_{h}^{\mathscr{L}_{2}} f(j h)=0$ для любого $j \in \mathbb{Z}$. Пусть $\varphi(x)=(1-\cos \alpha x) / \alpha^{2}$. Легко проверяется, что $\varphi(0)=\varphi^{\prime}(0)=0$, $\varphi^{\prime \prime}(0)=1$. Переходим к построению периодического локального тригонометрического сплайна $S$, соответствуюшего оператору $\mathscr{L}_{2}=\mathscr{D}^{2}+\alpha^{2} I$.

Для $x \in[j h ;(j+1) h], j \in \mathbb{Z}$, полагаем

$$
\begin{aligned}
S(x)=S(f, x)= & C_{0}^{(j)}+C_{1}^{(j)} \cos \alpha(x-j h) \\
& +C_{2}^{(j)} \sin \alpha(x-j h)+M^{(j)} \varphi\left(\left(x-\left(j+\frac{1}{2}\right) h\right)_{+}\right),
\end{aligned}
$$

где

$$
\varphi\left(x_{+}\right)= \begin{cases}\varphi(x), & x \geqslant 0 \\ 0, & x<0\end{cases}
$$


и

$$
\begin{aligned}
C_{0}^{(j)} & =\frac{\Delta_{h}^{\mathscr{L}_{2}} y_{j-1}}{4 \sin ^{2} \frac{\alpha h}{2} \cos \frac{\alpha h}{2}}, \\
C_{1}^{(j)} & =-\frac{y_{j+1}-2 y_{j}+y_{j-1}}{4 \sin ^{2} \frac{\alpha h}{2}}, \\
C_{2}^{(j)} & =\frac{y_{j+1}-y_{j-1}}{4 \sin \frac{\alpha h}{2} \cos \frac{\alpha h}{2}}, \\
M^{(j)} & =\frac{\alpha^{2}\left(\Delta_{h}^{\mathscr{L}_{2}} y_{j}-\Delta_{h}^{\mathscr{L}_{2}} y_{j-1}\right)}{4 \sin ^{2} \frac{\alpha h}{2} \cos \frac{\alpha h}{2}} .
\end{aligned}
$$

Из (2) следует, что на каждом из отрезков вида $[j h ;(j+1 / 2) h]$ и $[(j+1 / 2) h ;(j+1) h]$ тригонометрический сплайн $S(x)$ является линейной комбинацией трех функций: 1 , $\sin \alpha x, \cos \alpha x$. Функции $S(x)$ такого вида назьвают $L$-сплайнами второго порядка, соответствующими оператору $\mathscr{L}_{2}=\mathscr{D}^{2}+\alpha^{2} I$, с узлами в точках $\{j h\}_{j \in \mathbb{Z}}$ (основные уз-

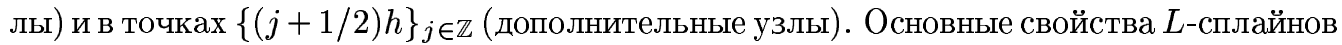
изложены, например, в монографии Дж. Алберга, Э. Нильсона и Дж. Уолша [3].

ТЕоРема 1. Пусть $h<\pi / \alpha$. Локальный прочесс аппроксимации (2)-(6) обладает следующими свойствами:

1) наследует локально свойство монотонности исходных данных $\left\{y_{j}\right\}, j \in \mathbb{Z}$, в том смысле, что

а) если $y_{j+1} \geqslant y_{j} \geqslant y_{j-1}\left(\right.$ или $\left.y_{j+1} \leqslant y_{j} \leqslant y_{j-1}\right)$, то функиия $S(x)$ при $x \in(j h ;(j+1 / 2) h)$ не убьвает (не возрастает);

б) если $y_{j+2} \geqslant y_{j+1} \geqslant y_{j}\left(\right.$ или $\left.y_{j+2} \leqslant y_{j+1} \leqslant y_{j}\right)$, то функиия $S(x)$ при $x \in((j+1 / 2) h ;(j+1) h)$ не убьвает (не возрастает);

2) наследует локально свойство $\left\{y_{j}\right\}$, близкое $\kappa$ выпуклости, а именно:

a) ecлu $\Delta_{h}^{\mathscr{L}_{2}} y_{j-1} \geqslant 0\left(\Delta_{h}^{\mathscr{L}_{2}} y_{j-1} \leqslant 0\right), m o^{\prime \prime}(x)+\alpha^{2} S(x) \geqslant 0\left(S^{\prime \prime}(x)+\alpha^{2} S(x) \leqslant 0\right)$ при $x \in(j h ;(j+1 / 2) h)$,

б) если $\Delta_{h}^{\mathcal{L}_{2}} y_{j} \geqslant 0\left(\Delta_{h}^{\mathcal{L}_{2}} y_{j} \leqslant 0\right)$, mo $S^{\prime \prime}(x)+\alpha^{2} S(x) \geqslant 0\left(S^{\prime \prime}(x)+\alpha^{2} S(x) \leqslant 0\right)$ npu $x \in((j+1 / 2) h ;(j+1) h)$;

3) $S(j h)=y_{j}+k \Delta_{h}^{\mathscr{L}_{2}} y_{j-1}(j \in \mathbb{Z})$, əде $k=\left(8 \cos \frac{\alpha h}{2} \cos ^{2} \frac{\alpha h}{4}\right)^{-1}$;

4) $S(x)$ имеет на всей оси $\mathbb{R}$ непрерьвную первую производную (т.е. $S^{\prime}(x) \in$ $C(\mathbb{R}))$, причем

$$
S^{\prime}(j h)=\frac{\alpha}{2 \sin \alpha h}\left(y_{j+1}-y_{j-1}\right)
$$

5) для любой функиии $f \in W_{\infty}^{\mathscr{L}_{2}}$ имеет место точное неравенство

$$
\left\|S^{\prime \prime}+\alpha^{2} S\right\|_{\infty} \leqslant \frac{1}{\cos \frac{\alpha h}{2}}
$$


ДокаЗАтельство. 1а) Пусть $x \in(j h ;(j+1 / 2) h)$, и пусть, например, $y_{j+1} \geqslant$ $y_{j} \geqslant y_{j-1}$. Из (2)-(5) выводим, что

$$
\begin{aligned}
S^{\prime}(x)= & \frac{\alpha}{4 \sin ^{2} \frac{\alpha h}{2} \cos \frac{\alpha h}{2}}\left[\left(y_{j+1}-y_{j}\right) \sin \alpha\left(x-j h+\frac{h}{2}\right)\right. \\
& \left.+\left(y_{j}-y_{j-1}\right) \sin \alpha\left(\frac{h}{2}-x+j h\right)\right] .
\end{aligned}
$$

Из этого равенства с учетом того, что $\alpha>0$ и $h<\pi / \alpha$, получаем, что $S^{\prime}(x) \geqslant 0$ при $x \in(j h ;(j+1 / 2) h)$, и поэтому на указанном промежутке функция $S(x)$ не убывает.

1б) Пусть теперь $x \in((j+1 / 2) h ;(j+1) h)$, и пусть, например, $y_{j+2} \geqslant y_{j+1} \geqslant y_{j}$. Из (2)-(6) получаем, что

$$
\begin{aligned}
S^{\prime}(x)= & \frac{\alpha}{4 \sin ^{2} \frac{\alpha h}{2} \cos \frac{\alpha h}{2}}\left[\left(y_{j+2}-y_{j+1}\right) \sin \alpha\left(x-\left(j+\frac{1}{2}\right) h\right)\right. \\
& \left.+\left(y_{j+1}-y_{j}\right) \sin \alpha\left(h-x+\left(j+\frac{1}{2}\right) h\right)\right] .
\end{aligned}
$$

Отсюда при $\alpha>0$ и $h<\pi / \alpha$ выводим, что $S^{\prime}(x) \geqslant 0$ при $x \in((j+1 / 2) h ;(j+1) h)$ и, следовательно, на этом промежутке функция $S(x)$ не убывает.

2) Применяя дифференциальньй оператор $\mathscr{D}^{2}+\alpha^{2} I$ к функции $S(x)$ на каждом из промежутков $(j h ;(j+1 / 2) h)$ и $((j+1 / 2) h ;(j+1) h)$, получаем, что

$$
S^{\prime \prime}(x)+\alpha^{2} S(x)= \begin{cases}A \cdot \Delta_{h}^{\mathscr{L}_{2}} y_{j-1}, & j h<x<(j+1 / 2) h, \\ A \cdot \Delta_{h}^{\mathscr{L}_{2}} y_{j}, & (j+1 / 2) h<x<(j+1) h,\end{cases}
$$

где $A=\alpha^{2}\left(4 \sin ^{2}(\alpha h / 2) \cos (\alpha h / 2)\right)^{-1}>0$. Отсюда следует утверждение пункта 2$)$.

3) Пусть $x \in[j h ;(j+1) h]$. Из (2)-(4) получаем

$$
S(j h)=C_{0}^{(j)}+C_{1}^{(j)}=\frac{y_{j+1}-(2 \cos \alpha h) y_{j}+y_{j-1}}{4 \sin ^{2} \frac{\alpha h}{2} \cos \frac{\alpha h}{2}}-\frac{y_{j+1}-2 y_{j}+y_{j-1}}{4 \sin ^{2} \frac{\alpha h}{2}} .
$$

После несложных преобразований $S(j h)$ можно записать в виде

$$
S(j h)=y_{j}+\frac{1}{8 \cos \frac{\alpha h}{2} \cos ^{2} \frac{\alpha h}{4}} \Delta_{h}^{\mathscr{L}_{2}} y_{j-1}
$$

Подставляя в $(2) x=(j+1) h$, аналогичным образом получаем, что

$$
S((j+1) h)=y_{j+1}+\frac{1}{8 \cos \frac{\alpha h}{2} \cos ^{2} \frac{\alpha h}{4}} \Delta_{h}^{\mathscr{L}_{2}} y_{j}
$$

и поэтому $S(x) \in C(\mathbb{R})$.

4) Непрерывность $S^{\prime}(x)$ во всех точках оси за исключением узлов сплайна $\{j h\}$ и $\{(j+1 / 2) h\}$ очевидна. Непрерывность $S^{\prime}(x)$ в точках $\{(j+1 / 2) h\}$ следует из того, что 
для функции $\varphi(x)=(1-\cos \alpha x) / \alpha^{2}$ справедливо равенство $\varphi^{\prime}(0)=0$. Воспользовавшись представлением для $S^{\prime}(x)$ из пункта 1$)$ теоремы 1 , получаем, что

$$
S^{\prime}(j h-0)=S^{\prime}(j h+0)=\frac{\alpha}{2 \sin \alpha h}\left(y_{j+1}-y_{j-1}\right)
$$

и, таким образом, $S^{\prime}(x) \in C(\mathbb{R})$.

5) Для доказательства последнего пункта теоремы 1 нужно, используя представление (7), оценить выражение $\left|\Delta_{h}^{\mathscr{L}_{2}} y_{j}\right|=\left|\Delta_{h}^{\mathscr{L}_{2}} f(j h)\right|$ для функций $f \in W_{\infty}^{\mathscr{L}_{2}}$. Вначале получим интегральное представление для разности $\Delta_{h}^{\mathscr{L}_{2}} f(j h)$. Не ограничивая общности, можно считать, что $j=0$. Любое решение линейного дифференциального уравнения $\mathscr{L}_{2}(\mathscr{D}) f=\left(\mathscr{D}^{2}+\alpha^{2} I\right) f=u, u \in L_{\infty}$, может быть записано в виде

$$
f(x)=C_{1} \cos \alpha(x-h)+C_{2} \sin \alpha(x-h)+\frac{1}{\alpha} \int_{h}^{x} u(t) \sin \alpha(x-t) d t,
$$

где $C_{1}$ и $C_{2}-$ произвольные константы. Отсюда следует, что

$$
\begin{gathered}
f(2 h)=C_{1} \cos \alpha h+C_{2} \sin \alpha h+\frac{1}{\alpha} \int_{h}^{2 h} u(t) \sin \alpha(2 h-t) d t \\
f(h)=C_{1}, \quad f(0)=C_{1} \cos \alpha h+C_{2} \sin \alpha h+\frac{1}{\alpha} \int_{0}^{h} u(t) \sin \alpha t d t
\end{gathered}
$$

где $u(t)=\mathscr{L}_{2}(\mathscr{D}) f(t)$. Поэтому из (1) имеем

$$
\begin{aligned}
\Delta_{h}^{\mathscr{L}_{2}} f(0) & =f(2 h)-(2 \cos \alpha h) f(h)+f(0) \\
& =\frac{1}{\alpha} \int_{h}^{2 h} u(t) \sin \alpha(2 h-t) d t+\frac{1}{\alpha} \int_{0}^{h} u(t) \sin \alpha t d t
\end{aligned}
$$

Поскольку для почти всех $t$ имеет место неравенство $\left|\mathscr{L}_{2}(\mathscr{D}) f(x)\right| \leqslant 1$, то из $(10)$ выводим оценку

$$
\left|\Delta_{h}^{\mathscr{L}_{2}} f(0)\right| \leqslant \frac{2}{\alpha} \int_{0}^{h} \sin \alpha t d t=\frac{4 \sin ^{2} \frac{\alpha h}{2}}{\alpha^{2}},
$$

которая является точной, причем знак равенства достигается для функций $f$ таких, что $\mathscr{L}_{2}(\mathscr{D}) f(x)=1$ для почти всех $t$ из отрезка $[0,2 h]$. Аналогично вьводится оценка и при других $j \in \mathbb{Z}$ :

$$
\sup _{j}\left|\Delta_{h}^{\mathscr{L}_{2}} f(j h)\right| \leqslant \frac{4 \sin ^{2} \frac{\alpha h}{2}}{\alpha^{2}} .
$$

Из (7) и (11) получаем точную оценку, сформулированную в пункте 5) теоремы 1:

$$
\left\|S^{\prime \prime}+\alpha^{2} S\right\|_{\infty} \leqslant A \cdot \frac{4 \sin ^{2} \frac{\alpha h}{2}}{\alpha^{2}}=\frac{1}{\cos \frac{\alpha h}{2}} .
$$

Теорема 1 полностью доказана. 


\section{2. Аппроксимация тригонометрическими сплайнами.}

ТЕОРема 2. При $h<\pi / \alpha$ справедливо равенство

$$
\sup _{f \in W_{\infty}^{\mathscr{L}_{2}}}\|f-S\|_{\infty}=\frac{1-\cos \frac{\alpha h}{2}}{\alpha^{2} \cos \frac{\alpha h}{2}}
$$

ДокАЗАТЕЛЬСтво. Получим интегральное представление для уклонения $f(x)-$ $S(x)$. Не нарушая общности, можно считать, что $x \in[h ; 2 h]$. Более того, можно также принять, что $x \in[h ; 3 h / 2]$, так как иначе можно сделать замену $x=1-v$. Для функции $f \in W_{\infty}^{\mathscr{L}_{2}}$ будем использовать представление (8). Сплайн $S(x)$ при $x \in[h ; 3 h / 2]$ имеет вид

$$
\begin{aligned}
S(x)= & \frac{\Delta_{h}^{\mathscr{L}_{2}} f(0)}{4 \sin ^{2} \frac{\alpha h}{2} \cos \frac{\alpha h}{2}}-\frac{\cos \alpha(x-h)}{4 \sin ^{2} \frac{\alpha h}{2}}[f(2 h)-2 f(h)+f(0)] \\
& +\frac{\sin \alpha(x-h)}{4 \sin \frac{\alpha h}{2} \cos \frac{\alpha h}{2}}[f(2 h)-f(0)] .
\end{aligned}
$$

Используя равенства (9) и (10), из формул (8) и (12) вьводим, что у разности $f(x)-$ $S(x)$ все неинтегральные слагаемые взаимно уничтожаются и при этом модуль уклонения $|f(x)-S(x)|$ имеет следующий вид:

$$
\begin{aligned}
|f(x)-S(x)|= & \mid \frac{1}{\alpha} \int_{h}^{x} u(t) \sin \alpha(x-t) d t \\
& -\left\{\frac{1}{4 \sin ^{2} \frac{\alpha h}{2} \cos \frac{\alpha h}{2}}\left[\frac{1}{\alpha} \int_{h}^{2 h} u(t) \sin \alpha(2 h-t) d t+\frac{1}{\alpha} \int_{0}^{h} u(t) \sin \alpha t d t\right]\right. \\
& -\frac{\cos \alpha(x-h)}{4 \sin ^{2} \frac{\alpha h}{2}}\left[\frac{1}{\alpha} \int_{h}^{2 h} u(t) \sin \alpha(2 h-t) d t+\frac{1}{\alpha} \int_{0}^{h} u(t) \sin \alpha t d t\right] \\
& \left.+\frac{\sin \alpha(x-h)}{4 \sin \frac{\alpha h}{2} \cos \frac{\alpha h}{2}}\left[\frac{1}{\alpha} \int_{h}^{2 h} u(t) \sin \alpha(2 h-t) d t-\frac{1}{\alpha} \int_{0}^{h} u(t) \sin \alpha t d t\right]\right\},
\end{aligned}
$$

где $u(t)=\mathscr{L}_{2}(\mathscr{D}) f(t)$. В формуле $(13)$ выражение под знаком модуля представим как сумму трех интегралов $J_{1}+J_{2}+J_{3}$, где

$$
\begin{aligned}
& J_{1}=\int_{0}^{h} L_{1}(x, t) u(t) d t, \\
& J_{2}=\int_{x}^{2 h} L_{2}(x, t) u(t) d t, \\
& J_{3}=\int_{h}^{x} L_{3}(x, t) u(t) d t,
\end{aligned}
$$


и

$$
\begin{aligned}
& L_{1}(x, t)=-\frac{\sin \alpha t}{4 \alpha \sin \frac{\alpha h}{2}}\left[\frac{1}{\cos \frac{\alpha h}{2} \sin \frac{\alpha h}{2}}-\frac{\cos \alpha(x-h)}{\sin \frac{\alpha h}{2}}-\frac{\sin \alpha(x-h)}{\cos \frac{\alpha h}{2}}\right] \\
& =-\frac{\sin \alpha t\left(1-\cos \alpha\left(x-\frac{3 h}{2}\right)\right)}{4 \alpha \sin ^{2} \frac{\alpha h}{2} \cos \frac{\alpha h}{2}}, \\
& L_{2}(x, t)=-\frac{\sin \alpha(2 h-t)}{4 \alpha \sin \frac{\alpha h}{2}}\left[\frac{1}{\cos \frac{\alpha h}{2} \sin \frac{\alpha h}{2}}-\frac{\cos \alpha(x-h)}{\sin \frac{\alpha h}{2}}+\frac{\sin \alpha(x-h)}{\cos \frac{\alpha h}{2}}\right] \\
& =-\frac{\sin \alpha(2 h-t)}{4 \alpha \sin ^{2} \frac{\alpha h}{2} \cos \frac{\alpha h}{2}}\left(1-\cos \alpha\left(x-\frac{h}{2}\right)\right) \text {, } \\
& L_{3}(x, t)=\frac{1}{\alpha}\left\{\sin \alpha(x-t)-\left[\frac{1}{4 \cos \frac{\alpha h}{2} \sin ^{2} \frac{\alpha h}{2}}-\frac{\cos \alpha(x-h)}{4 \sin ^{2} \frac{\alpha h}{2}}\right.\right. \\
& \left.\left.+\frac{\sin \alpha(x-h)}{4 \sin \frac{\alpha h}{2} \cos \frac{\alpha h}{2}}\right] \sin \alpha(2 h-t)\right\} \\
& =\frac{1}{\alpha}\left[\sin \alpha(x-t)-\frac{\sin \alpha(2 h-t)}{4 \sin ^{2} \frac{\alpha h}{2} \cos \frac{\alpha h}{2}}\left(1-\cos \alpha\left(x-\frac{h}{2}\right)\right)\right] .
\end{aligned}
$$

Из (17) и (18) легко следует, что при $\alpha>0, h<\pi / \alpha L_{1}(x, t) \leqslant 0$ при $0 \leqslant t \leqslant h$ и $L_{2}(x, t) \leqslant 0$ при $h \leqslant x \leqslant t \leqslant 2 h$.

Докажем теперь, что $L_{3}(x, t) \leqslant 0$ при $h \leqslant t \leqslant x \leqslant 3 h / 2$. Функция $L_{3}(x, t)$, как функция от $t$, может быть записана в виде

$$
L_{3}(x, t)=A_{1}(x) \sin \alpha t+A_{2}(x) \cos \alpha t,
$$

поэтому она сохраняет знак на интервалах длины $\pi / \alpha$. Следовательно, для доказательства неравенства $L_{3}(x, t) \leqslant 0$ при $h \leqslant t \leqslant x$ в силу того, что $x-h \leqslant h / 2<\pi /(2 \alpha)$, достаточно показать, что $L_{3}(x, h) \leqslant 0$ и $L_{3}(x, x) \leqslant 0$ при $h \leqslant x \leqslant 3 h / 2$. В самом деле,

$$
L_{3}(x, h)=\frac{\cos \alpha\left(x-\frac{3 h}{2}\right)-1}{2 \alpha \sin \frac{\alpha h}{2}} \leqslant 0
$$

так как $\alpha>0$ и $0<h<\pi / \alpha$ и

$$
L_{3}(x, x)=\frac{\cos \alpha\left(x-\frac{3 h}{2}\right)-1}{4 \alpha \sin ^{2} \frac{\alpha h}{2} \cos \frac{\alpha h}{2}} \sin \alpha(2 h-x) \leqslant 0
$$

при $0<h<\pi / \alpha$ и $h \leqslant x \leqslant 3 h / 2$.

Из доказанных неравенств $L_{j}(x, t) \leqslant 0, j=1,2,3$ (для соответствующих значений аргументов), и формул (13)-(16) следует, что для получения оценки сверху для величины погрешности $|f(x)-S(x)|$ на классе $W_{\infty}^{\mathscr{L}_{2}}$ в формуле $(13)$ функцию $u(t)=\mathscr{L}_{2}(\mathscr{D}) f(t)$ можно заменить на 1 . Вычисляя при этом интегралы $J_{1}, J_{2}$ и $J_{3}$, получаем

$$
|f(x)-S(x)| \leqslant\left|J_{1}+J_{2}+J_{3}\right|=\frac{1-\cos \frac{\alpha h}{2}}{\alpha^{2} \cos \frac{\alpha h}{2}} .
$$

Неравенство (20) является точньм, причем знак равенства достигается, например, для функций $f$ таких, что $\mathscr{L}_{2}(\mathscr{D}) f(t)=1$ при почти всех $t \in[0,2 h]$. Тем самым, доказано равенство

$$
\sup _{f \in W_{\infty}^{\mathscr{L}_{2}}}\|f-S\|_{\infty}=\frac{1-\cos \frac{\alpha h}{2}}{\alpha^{2} \cos \frac{\alpha h}{2}} .
$$


ЗАмЕЧАнИЕ. Изпункта 3) теоремы 1 и неравенства (11) для любой функции $f \in W_{\infty}^{\mathscr{L}_{2}}$ получаем оценку

$$
\begin{aligned}
|f(j h)-S(j h)| & \leqslant \frac{1}{8 \cos \frac{\alpha h}{2} \cos ^{2} \frac{\alpha h}{4}} \cdot \sup _{j}\left|\Delta_{h}^{\mathscr{L}_{2}} y_{j}\right| \\
& \leqslant \frac{1}{8 \cos \frac{\alpha h}{2} \cos ^{2} \frac{\alpha h}{4}} \cdot \frac{4 \sin ^{2} \frac{\alpha h}{2}}{\alpha^{2}}=\frac{1-\cos \frac{\alpha h}{2}}{\alpha^{2} \cos \frac{\alpha h}{2}} .
\end{aligned}
$$

Полученное неравенство является точным. Сопоставляя его с утверждением теоремы 2 , заключаем, что точки $\{j h\}$ (основные узлы сплайна) для некоторых функций $f \in W_{\infty}^{\mathscr{L}_{2}}$ могут являться точками максимального уклонения в метрике пространства $C$ тригонометрического сплайна $S$ от функции $f$. Это свойство было для нас ключевым при построении формул (2)-(6).

3. Связь с поперечниками. Пусть, как обычно,

$$
d_{n}\left(W_{\infty}^{\mathscr{L}_{2}}\right)_{\infty}=\inf _{L_{n}: \operatorname{dim} L_{n} \leqslant n} \sup _{f \in W_{\infty}^{\mathscr{L}_{2}}} \inf _{\varphi \in L_{n}}\|f-\varphi\|_{\infty}
$$

и

$$
K_{n}\left(W_{\infty}^{\mathscr{L}_{2}}, B W_{\infty}^{\mathscr{L}_{2}}\right)_{\infty}=\inf _{L_{n}: \operatorname{dim} L_{n} \leqslant n} \sup _{f \in W_{\infty}^{\mathscr{L}_{2}}} \inf _{\varphi \in L_{n} \cap B W_{\infty}^{\mathscr{L}_{2}}}\|f-\varphi\|_{\infty}
$$

- соответственно $n$-й поперечник по Колмогорову и $n$-й поперечник по Коновалову (его еше назьвают относительньм поперечником) класса $W_{\infty}^{\mathscr{L}_{2}}$ в равномерной метрике. Здесь $\mathscr{L}_{2}=\mathscr{L}_{2}(\mathscr{D})=\mathscr{D}^{2}+\alpha^{2} I, \alpha>0, B>0$ и внешний inf берется по всем подпространствам $L_{n}$ размерности не вьше $n$. Из определений следует, что если $B_{1} \geqslant B_{2}$, то

$$
d_{n}\left(W_{\infty}^{\mathscr{L}_{2}}\right)_{\infty} \leqslant K_{n}\left(W_{\infty}^{\mathscr{L}_{2}}, B_{1} W_{\infty}^{\mathscr{L}_{2}}\right)_{\infty} \leqslant K_{n}\left(W_{\infty}^{\mathscr{L}_{2}}, B_{2} W_{\infty}^{\mathscr{L}_{2}}\right)_{\infty}
$$

Подпространства функций, реализующие внешний inf в этих определениях, назьваются экстремальным.м. Не вдаваясь в обширную библиографию по поперечникам функциональных классов в различных пространствах, отметим только точные результаты в периодическом случае (период функций равен $2 \pi$ ), связанные с оператором $\mathscr{L}_{2}=\mathscr{D}^{2}+\alpha^{2} I$.

В 1938 году М. Г. Крейн [5] при $m>\alpha$ вычислил $E_{m-1}\left(W_{\infty}^{\mathscr{L}_{2}}\right)_{\infty}$ - величину наилучшего приближения класса $W_{\infty}^{\mathscr{L}_{2}}$ в равномерной метрике пространством тригонометрических многочленов порядка $m-1$. Величина $E_{m-1}\left(W_{\infty}^{\mathscr{L}_{2}}\right)_{\infty}$ дает точную оценку сверху для нечетного поперечника $d_{2 m-1}\left(W_{\infty}^{\mathscr{L}_{2}}\right)_{\infty}$. Такая же оценка снизу для указанного поперечника при $m \geqslant 2 \alpha$ получена В. Т. Шевалдиным [6]. Точная оценка сверху для четного поперечника $d_{2 m}\left(W_{\infty}^{\mathscr{L}_{2}}\right)_{\infty}$ может быть также выведена из работы С.И. Новикова [7], которьй при $m \geqslant 2 \alpha$ оценил в каждой точке уклонение функций $f \in W_{\infty}^{\mathscr{L}_{2}}$ от интерполяционных тригонометрических сплайнов с $2 m$ равномерными узлами на периоде и вычислил величину этого уклонения на классе $W_{\infty}^{\mathscr{L}_{2}}$. Точную оценку снизу для четного поперечника $d_{2 m}\left(W_{\infty}^{\mathscr{L}_{2}}\right)_{\infty}$ при $m>\alpha$ получила И. Н. Володина [8], используя один общий прием В. М. Тихомирова. Подчеркиваем, что во всех перечисленных работах рассматривались функции периода $2 \pi$, и главный результат, вытекающий из этих работ, состоял в следующем равенстве, справедливом при $m>\alpha$ :

$$
d_{2 m-1}\left(W_{\infty}^{\mathscr{L}_{2}}\right)_{\infty}=d_{2 m}\left(W_{\infty}^{\mathscr{L}_{2}}\right)_{\infty}=\frac{2}{\pi} \max _{t}\left|\sum_{s \in \mathbb{Z}} \frac{e^{i(2 s+1) \pi t}}{(2 s+1)\left(\alpha^{2}-(2 s+1)^{2} m^{2}\right)}\right|
$$


В нашей работе мы имеем дело с функциями периода 1. Пересчитьвая в этом случае величины поперечников (например, с помощью формул из § 2 и 55 работы [9]), выводим, что для 1-периодических функций при $m>\alpha /(2 \pi)$ справедливо следующее равенство:

$$
d_{2 m-1}\left(W_{\infty}^{\mathscr{L}_{2}}\right)_{\infty}=d_{2 m}\left(W_{\infty}^{\mathscr{L}_{2}}\right)_{\infty}=\frac{1-\cos \frac{\alpha}{4 m}}{\alpha^{2} \cos \frac{\alpha}{4 m}}
$$

причем экстремальными подпространствами для четного поперечника $d_{2 m}\left(W_{\infty}^{\mathscr{L}_{2}}\right)_{\infty}$ являются пространство тригонометрических полиномов степени $m-1$ и пространство интерполящионных тригонометрических сплайнов с $2 m$ равномерными узлами на периоде длины 1 (при $m \geqslant \alpha / \pi)$.

Отметим, что для класса 1-периодических функций

$$
W_{\infty}^{r}=\left\{f: f^{(r-1)} \in A C,\left\|f^{(r)}\right\|_{\infty} \leqslant 1\right\}, \quad r \in \mathbb{N}, \quad r \geqslant 3,
$$

Ю.Н. Субботин и С. А. Теляковский [10], [11] доказали, что неравенство

$$
B \geqslant \frac{4}{\pi^{2}} \log (\min (n, r))+A
$$

где $A$ - некоторая константа, гарантирует равенство величин поперечников по Коновалову $K_{n}\left(W_{\infty}^{r}, B W_{\infty}^{r}\right)_{\infty}$ и по Колмогорову $d_{n}\left(W_{\infty}^{r}\right)_{\infty}$. Еще ранее Субботин [1] показал, что при $B \geqslant 1$ справедливо равенство

$$
K_{2 n}\left(W_{\infty}^{2}, B W_{\infty}^{2}\right)_{\infty}=d_{2 n}\left(W_{\infty}^{2}\right)_{\infty}=\left(32 n^{2}\right)^{-1} .
$$

Tеорема 3. Пусmь $h=1 /(2 m), m>\alpha /(2 \pi) u B \geqslant 1 / \cos (\alpha h / 2)$. Тогдa

$$
K_{2 m}\left(W_{\infty}^{\mathscr{L}_{2}}, B W_{\infty}^{\mathscr{L}_{2}}\right)_{\infty}=\frac{1-\cos \frac{\alpha}{4 m}}{\alpha^{2} \cos \frac{\alpha}{4 m}}
$$

ДокАЗАТЕЛЬСтво. Оценка снизу для поперечника по Коновалову следует из неравенства

$$
d_{2 m}\left(W_{\infty}^{\mathscr{L}_{2}}\right)_{\infty} \leqslant K_{2 m}\left(W_{\infty}^{\mathscr{L}_{2}}, B W_{\infty}^{\mathscr{L}_{2}}\right), \quad B>0
$$

и равенства (21). Для оценки сверху воспользуемся результатами теорем 1 и 2 . Из равенств (2)-(6) получаем, что если считать $\left\{y_{j}\right\}$ произвольной последовательностью действительных чисел, удовлетворяющей условию $y_{j+n}=y_{j}, j \in \mathbb{Z}$, то при четном $n=2 m$ сплайн $S(x)$ является линейной комбинацией вида

$$
S(x)=\sum_{j=0}^{2 m-1} y_{j} \theta_{j}(x)
$$

где $\theta_{j}(x)$ - некоторые гладкие 1-периодические функции. Очевидно, что размерность подпространства указанных сплайнов не вьше $2 m$. Поэтому из пункта 5 ) теоремы 1 и теоремы 2 при $h=1 /(2 m), m>\alpha /(2 \pi)$ и $B \geqslant B_{1}=1 / \cos (\alpha h / 2)$ в силу определения относительного поперечника получаем

$$
K_{2 m}\left(W_{\infty}^{\mathscr{L}_{2}}, B W_{\infty}^{\mathscr{L}_{2}}\right)_{\infty} \leqslant K_{2 m}\left(W_{\infty}^{\mathscr{L}_{2}}, B_{1} W_{\infty}^{\mathscr{L}_{2}}\right)_{\infty} \leqslant \sup _{f \in W_{\infty}^{\mathscr{L}_{2}}}\|f-S\|_{\infty}=\frac{1-\cos \frac{\alpha}{4 m}}{\alpha^{2} \cos \frac{\alpha}{4 m}}
$$

Теорема 3 доказана.

ЗАмЕчАниЕ. Линейный локальньй процесс аппроксимации (2)-(6) порождает еще одно экстремальное (в смысле поперечника по Колмогорову) подпространство тригонометрических сплайнов с равномерными узлами, причем эти сплайны не являются интерполящионньми. 


\section{СПИСОК ЦИТИРОВАННОЙ ЛИТЕРАТУРЫ}

[1] Субботин Ю.Н. Наследование свойств монотонности и выпуклости при локальной аппроксимации // ЖВМиМФ. 1993. Т. 33. № 7. С. 996-1003.

[2] Субботин Ю. Н., Черных Н. И. Порядок наилучшей сплайн-аппроксимации некоторых классов функций // Матем. заметки. 1970. Т. 7. №1. С. 31-42.

[3] Алберг Дж., Нильсон Э., Уолш Дж. Теория сплайнов и ее приложения. М.: Мир, 1972.

[4] Шевалдин В. Т. Истокообразные сплайны и поперечники классов периодических функций. Дисс. ... д. ф.--м. н. Екатеринбург: ИММ УрО РАН, 1996.

[5] Крейн М.Г. К теории наилучшего приближения периодических функций // Докл. АН CCCP. 1938. T. 18. № 4, 5. C. 603-607.

[6] Шевалдин В. Т. $L$-сплайны и поперечники // Матем. заметки. 1983. Т. 33. № 5. С. $735-744$.

[7] Новиков С. И. Приближение класса $W_{\infty}^{\mathscr{L}_{n}}$ интерполяционньми периодическими сплайнами // Приближение функций полиномами и сплайнами. Свердловск: УНЦ АН СССР, 1985. C. $118-126$.

[8] Volodina I. N. Exact value of widths of certain class of solutions of linear differential equations // Analysis Math. 1985. V. 11. № 1. P. 85-92.

[9] Шевалдин В. Т. Некоторые задачи экстремальной интерполяции в среднем для линейных дифференциальных операторов // Тр. МИАН. 1983. Т. 164. С. 203-240.

[10] Субботин Ю.Н., Теляковский С. А. Асимптотика констант Лебега для периодических интерполяционных сплайнов с равномерными узлами // Матем. сб. 2000. Т. 191. № 8. C. 131-140.

[11] Subbotin Yu. N., Telyakovskii S. A. Splines and relative widths of classes of differentiable functions // Proc. of the Steklov Institute of Math., Suppl. 1. 2001. P. 225-234.

(К. В. Костоусов) Уральский государственный университет

Поступило им. А. М. Горького, г. Екатеринбург 01.07 .2003

(В. Т. Шевалдин) Институт математики и механики УрО РАН, г. Екатеринбург

E-mail: Valerii.Shevaldin@imm.uran.ru 